\title{
DNA methylation age acceleration is associated with ALS age of onset and survival
}

\author{
Ming Zhang ${ }^{1,2,3,4} \cdot$ Paul M. McKeever ${ }^{2,5} \cdot$ Zhengrui Xi $^{3,6} \cdot$ Danielle Moreno $^{2} \cdot$ Christine Sato $^{2} \cdot$ Tessa Bergsma $^{2}$. \\ Philip McGoldrick ${ }^{2,5}$. Julia Keith ${ }^{5,7}$. Janice Robertson ${ }^{2,5} \cdot$ Lorne Zinman $^{7,8}$ • Ekaterina Rogaeva ${ }^{2,8}$
}

Received: 28 November 2019 / Revised: 27 January 2020 / Accepted: 28 January 2020 / Published online: 7 March 2020

(c) The Author(s) 2020

Amyotrophic lateral sclerosis (ALS) patients, including C9orf72-carriers and identical twins [1], have highly variable disease characteristics (e.g., duration and age/site of onset) [7], suggesting the influence of epigenetic variations. DNA methylation (DNAm) is a key epigenetic modification and linked to the risk of several neurodegenerative diseases (Supplementary introduction). The cumulative assessment of DNAm levels at age-related CpGs allows the estimation of multi-tissue DNAm age (epigenetic clock), which could be more accurate for assessing biological age than chronological age. Hypermethylation of the CpG-island 5' of the C9orf72-repeat and DNAm-age acceleration have been reported to be associated with $C 9$ orf 72 -disease duration and age of onset $[9,10]$. However, epigenetic modifiers in genetically unexplained ALS patients are largely unknown.

Hence, we conducted a genome-wide investigation of DNAm (Infinium MethylationEPIC chip) in samples from blood $(n=249)$ or tissues from the central nervous system (CNS) $(n=18)$ of mainly sporadic ALS patients (Table 1$)$, and assessed the association of DNAm-age acceleration with

Electronic supplementary material The online version of this article (https://doi.org/10.1007/s00401-020-02131-z) contains supplementary material, which is available to authorized users.

Ming Zhang

mingzhang@tongji.edu.cn

$\triangle$ Lorne Zinman

Lorne.Zinman@sunnybrook.ca

$\triangle$ Ekaterina Rogaeva

ekaterina.rogaeva@utoronto.ca

1 Shanghai First Rehabilitation Hospital, School of Medicine, Tongji University, Shanghai 200090, China

2 Tanz Centre for Research in Neurodegenerative Diseases, University of Toronto, 60 Leonard Ave., Toronto, ON M5T 2S8, Canada

3 Clinical Center for Brain and Spinal Cord Research, Tongji University, Shanghai 200092, China disease age of onset and survival (Supplementary methods). Considering the reported 3-year error for estimating DNAm age, we classified the patients into three groups: normal aging $(n=82$, DNAm-age acceleration between -3 and 3 years, median $=0.5$ years $)$, slow aging $(n=125$, DNAm-age acceleration $<-3$ years, median $=-6.3$ years), and fast aging $(n=42$, DNAm-age acceleration $>3$ years, median $=5.7$ years).

Multivariate linear regression analysis detected a highly significant association between blood-based DNAm-age acceleration and ALS age of onset (adjusted $p$ value $=2.2 \mathrm{E}$ $16, B=-1.29, R^{2}=0.33, n=249$ ) (Supplementary methods, Fig. 1a), suggesting that for every 5 -year increase in DNAm-age acceleration there is a 6.4-year earlier onset. Cox proportional hazard regression analysis revealed that a faster DNAm-age acceleration is significantly associated with an earlier age of onset [adjusted $p$ value $=9.3 \mathrm{E}-16$, hazard ratio $(\mathrm{HR})=2.12 ; 95 \%$ confidence interval $(\mathrm{CI}): 1.76-2.54]$, suggesting that the hazard increased by $112 \%$ (Fig. 1c). The median age of onset in the fast aging group (48 years; 95\%CI: 42-51) was 18 years younger than the slow aging group (66 years; 95\%CI: 63-68). Significant results were not

Institute for Advanced Study, Tongji University, Shanghai, China

5 Department of Laboratory Medicine and Pathobiology, University of Toronto, 27 King's College Circle, Toronto, ON M5S 1A1, Canada

6 Shanghai East Hospital, School of Medicine, Tongji University, Shanghai 200120, China

7 Sunnybrook Health Sciences Centre, 2075 Bayview Ave, Toronto, ON M4N 3M5, Canada

8 Division of Neurology, Department of Medicine, University of Toronto, Toronto, Canada 
Table 1 Sample characteristics of the ALS cohort

\begin{tabular}{lll}
\hline Characteristics of ALS patients & Blood & CNS cohort $^{\text {a }}$ \\
\hline Sporadic ALS cases $(n)$ & 200 & 17 \\
Familial ALS cases $(n)$ & 49 & 1 \\
Sex (male, $n \%$ ) & $148,59.4 \%$ & $13,72.2 \%$ \\
Age of onset (years) & & \\
$\quad$ Mean & 59 & 59 \\
Range & $16-87$ & $31-79$ \\
Site of onset (n, $\%)$ & & $4,22.2 \%$ \\
Bulbar & $67,27 \%$ & $14,77.8 \%$ \\
Limb & $172,73 \%$ & \\
\hline
\end{tabular}

${ }^{a}$ Frontal cortex and cervical spinal cord from the central nervous system (CNS)

driven by subgroups stratified by sex or site of onset (Figs. S2-S3, Fig. S6).

Furthermore, Cox proportional hazard regression analysis revealed that a faster DNAm-age acceleration was significantly associated with a shorter survival with an increased hazard of $107 \%$ (adjusted $p$ value $=1.7 \mathrm{E}-7, \mathrm{HR}=2.07$; 95\%CI: 1.58-2.72) (Supplementary methods, Fig. 1d). The median survival age was 10 years shorter in the fast aging group (65 years; 95\%CI: 54-70) than the slow aging group (75 years; 95\%CI: 74-77). We replicated this observation by re-analyzing DNAm data from our previous study of 30 C9orf72 patients [10], and found that the median survival age was 15 years shorter in the fast vs slow aging group (adjusted $p$ value $=0.01, \mathrm{HR}=3.5 ; 95 \% \mathrm{CI}: 1.3-9.3)$, suggesting that the hazard increased by $250 \%$ (Fig. S1). The significant findings were not driven by subgroups stratified by sex or site of onset (Fig. S4-S6).

Previously, we reported that CNS-based DNAm-age acceleration is significantly associated with $C 9$ orf 72 disease age of onset and duration, using DNA from frontal cortex (FC) and cervical spinal cord (CSC) [10]. To evaluate this association in genetically unexplained ALS patients, we investigated DNA isolated from FC and CSC tissues of 18 ALS cases. Even with the modest CNS sample size, DNAm-age acceleration is associated with age of onset in DNA from FC (adjusted $p$ value $=8.4 \mathrm{E}-4$, $\mathrm{B}=-1.73, R^{2}=0.48$ ); and CSC (adjusted $p$ value $=3.7 \mathrm{E}$ $\left.5, \mathrm{~B}=-1.69, R^{2}=0.65\right)$ (Fig. 1b). The linear regression model suggests that a 5-year increase in CNS-based DNAm-age acceleration is linked to an 8.4-8.7 year earlier onset. Furthermore, the Cox proportional hazard regression analysis suggests that CNS-based DNAm-age acceleration is significantly associated with survival (FC: $p$ value $=0.006$; CSC: $p$ value $=0.0009)($ Fig. S7). As expected for a multi-tissue epigenetic clock, there was no significant difference in DNAm-age acceleration between the two CNS tissues ( $p$ value $=0.13$; Fig. S8). Since ALS is often associated with mixed pathologies [2], we evaluated if FC pathology (tau or TDP-43 inclusions, Table S1) may affect our findings. However, a subgroup analysis of patients with $(n=5)$ or without $(n=13)$ FC pathology found no significant difference in DNAm-age acceleration ( $p$ value $=0.78$, Fig. S9)

Locus-by-locus analysis of blood DNAm levels of 835,424 CpGs (not overlapping known genetic polymorphisms) found no CpGs associated with age of onset at the genome-wide significant level in 249 ALS patients (Fig. S10, Table S2-S3).

Cumulatively, our study revealed that blood/CNS-based DNAm-age acceleration is significantly associated with age of onset and survival in genetically unexplained ALS patients, suggesting a novel epigenetic modifier. In C9orf 72 patients, we observed a trend for a stronger association of survival with DNAm-age acceleration vs a general ALS cohort, but with a much broader 95\%CI (likely due to the modest size of the $C 9$ orf 72 cohort; $n=30$ ). DNAm-age acceleration was comparable between the two investigated CNS tissues; however, we observed a tendency for a stronger association of DNAm-age acceleration with age of onset in CNS vs non-autopsy blood samples. Both trends should be clarified by analyzing a larger sample set in the future (Supplementary discussion).

Our findings strongly support the use of DNAm-age acceleration as a biomarker of biological aging. The epigenetic clock may also be used to stratify results obtained in clinical trials (e.g., response to treatment in the fast vs slow aging groups). Notably, a recent pilot study reported using the epigenetic clock in a clinical trial intended to regenerate the thymus to prevent signs of immunosenescence [3], encouraging the use of the epigenetic clock to estimate the effectiveness of aging interventions.

The molecular mechanism underlying the epigenetic clock and its link to disease presentation is largely unknown. Age-related CpGs are mapped to promoter, enhancer and polycomb protein-binding regions [4]. Aberrant DNAm in these regions may regulate gene expression involved in key biological processes (cell growth/proliferation and death/ survival [5]). Future studies could also clarify the association between DNAm-age acceleration and other ALS phenotypes (e.g., severity), or in ALS patients with other mutations (e.g., in SOD1), and different neurodegenerative phenotypes in the longitudinal Genetic Frontotemporal dementia Initiative cohort [6] and the Dominantly inherited Alzheimer's disease cohort [8] (Supplementary discussion).

Hence, DNAm-age acceleration may be used as an aging biomarker in future clinical trials to predict the effect of drugs on modulating ALS age of onset or survival. 

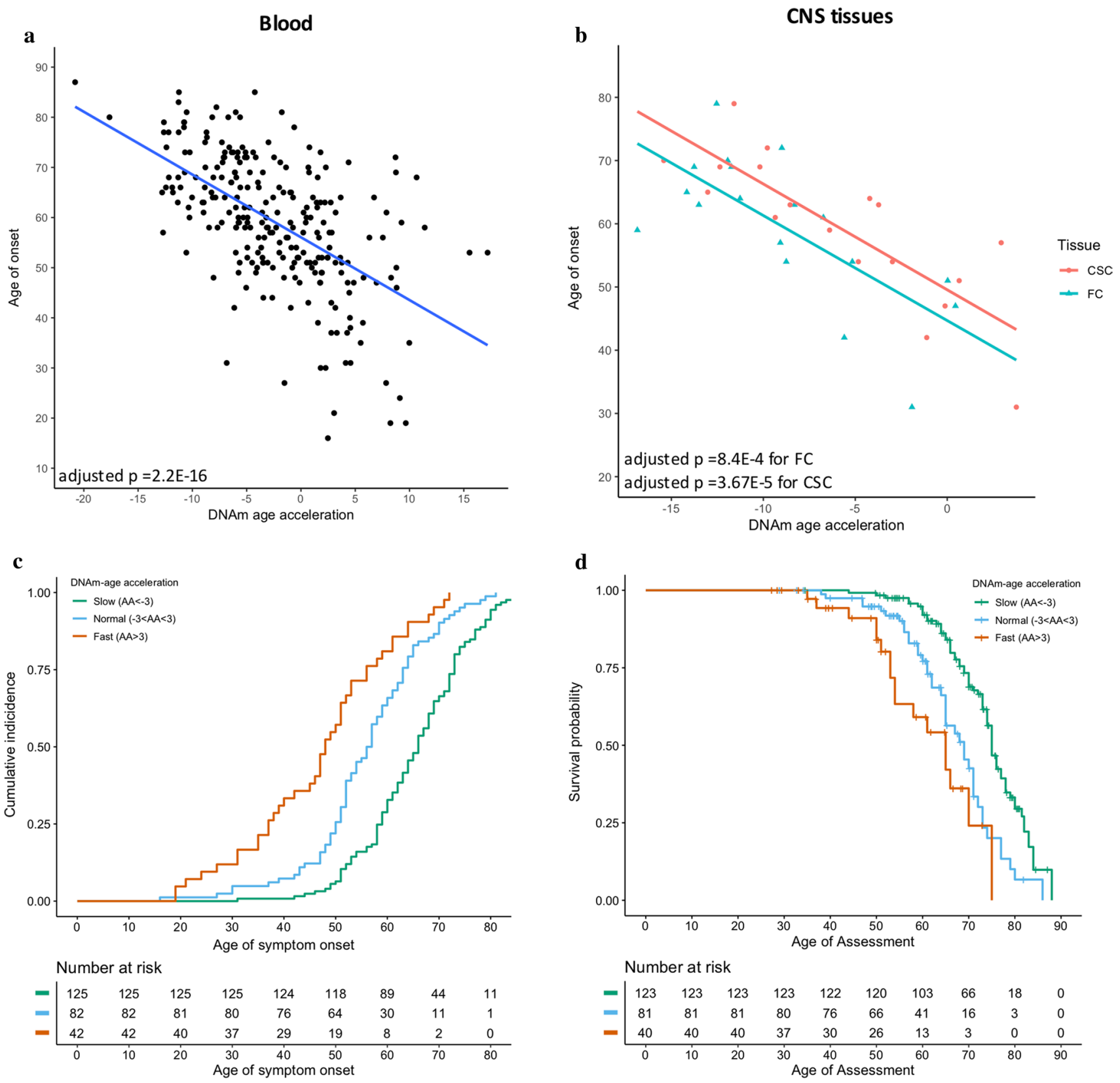

Fig. 1 DNAm-age acceleration is significantly associated with ALS age of onset and survival. a Scatter plot showing the association between blood-based DNAm-age acceleration and age of onset (adjusted $p$ value $=2.2 \mathrm{E}-16, B=-1.29, R^{2}=0.33$; blood cell abundance adjusted $p$ value $\left.=2 \mathrm{E}-16, B=-1.22, R^{2}=0.47, n=249\right)$. The solid line represents the linear regression trend. b Scatter plot showing the association between CNS-based DNAm-age acceleration and age of onset. Tissues were obtained from 18 ALS cases: frontal cortex (FC) (adjusted $p$ value $=8.4 \mathrm{E}-4, B=-1.73, R^{2}=0.48$ ), and cervical spinal cord (CSC) (adjusted $p$ value $=3.67 \mathrm{E}-5, B=-$ $\left.1.69, R^{2}=0.65\right)$. The solid line represents the linear regression trend. c Kaplan-Meier curve of cumulative incidence of ALS age

Acknowledgements This work was in part supported by the ALS Canada (ER, LZ, MZ), Canadian Consortium on Neurodegeneration in Aging (ER, MZ), ALS Canada-Brain Canada Hudson Translational Team Grant (JR, LZ, ER), the Shanghai Pujiang Program 19PJ1410300

of onset ( $n=249)$ stratified into three groups based on blood-based DNAm-age acceleration (adjusted $p$ value $=9.3 \mathrm{E}-16, \quad \mathrm{HR}=2.12$; 95\%CI: $1.76-2.54$; blood cell abundance adjusted $p$ value $=5.3 \mathrm{E}$ 12 , $\mathrm{HR}=2.39$, 95\% CI: 1.86-3.06). d Kaplan-Meier curve of survival probability $(n=244)$ stratified into three aging groups based on blood-based DNAm-age acceleration (adjusted $p$ value $=1.7 \mathrm{E}$ 7, $\mathrm{HR}=2.07$; 95\% CI: 1.58-2.72; blood cell abundance adjusted $p$ value $=4.4 \mathrm{E}-8, \mathrm{HR}=2.21,95 \% \mathrm{CI}: 1.66-2.93$ ). Slow aging: DNAmage acceleration $<-3$ years, normal aging: DNAm-age acceleration between -3 and 3 years, and fast aging: DNAm-age acceleration $>3$ years. AA represents DNAm-age acceleration

(MZ), Milton Safenowitz Postdoctoral Fellowship from the American ALS Association (PMM), and James Hunter ALS Initiative (LZ, JR); JR holds the James Hunter Family Chair in ALS Research. 


\section{Compliance with ethical standards}

Conflict of interest The authors report no competing interests.

Open Access This article is licensed under a Creative Commons Attribution 4.0 International License, which permits use, sharing, adaptation, distribution and reproduction in any medium or format, as long as you give appropriate credit to the original author(s) and the source, provide a link to the Creative Commons licence, and indicate if changes were made. The images or other third party material in this article are included in the article's Creative Commons licence, unless indicated otherwise in a credit line to the material. If material is not included in the article's Creative Commons licence and your intended use is not permitted by statutory regulation or exceeds the permitted use, you will need to obtain permission directly from the copyright holder. To view a copy of this licence, visit http://creativecommons.org/licenses/by/4.0/.

\section{References}

1. Al-Chalabi A, Fang F, Hanby MF, Leigh PN, Shaw CE, Ye W et al (2010) An estimate of amyotrophic lateral sclerosis heritability using twin data. J Neurol Neurosurg Psychiatry 81:1324-1326. https://doi.org/10.1136/jnnp.2010.207464

2. Alafuzoff I, Kovacs GG (2017) Comorbidities. Handb Clin Neurol 145:573-577. https://doi.org/10.1016/B978-0-12-802395-2.00036 $-5$

3. Fahy GM, Brooke RT, Watson JP, Good Z, Vasanawala SS, Maecker $\mathrm{H}$ et al (2019) Reversal of epigenetic aging and immunosenescent trends in humans. Aging Cell 18:e13028. https://doi. org/10.1111/acel.13028

4. Field AE, Robertson NA, Wang T, Havas A, Ideker T, Adams PD (2018) DNA methylation clocks in aging: categories, causes, and consequences. Mol Cell 71:882-895. https://doi.org/10.1016/j. molcel.2018.08.008

5. Horvath S (2013) DNA methylation age of human tissues and cell types. Genome Biol 14:R115. https://doi.org/10.1186/ gb-2013-14-10-r115

6. Rohrer JD, Nicholas JM, Cash DM, van Swieten J, Dopper E, Jiskoot L et al (2015) Presymptomatic cognitive and neuroanatomical changes in genetic frontotemporal dementia in the Genetic Frontotemporal dementia Initiative (GENFI) study: a cross-sectional analysis. Lancet Neurol 14:253-262. https://doi. org/10.1016/S1474-4422(14)70324-2

7. Swinnen B, Robberecht W (2014) The phenotypic variability of amyotrophic lateral sclerosis. Nat Rev Neurol 10:661-670. https ://doi.org/10.1038/nrneurol.2014.184

8. Tang M, Ryman DC, McDade E, Jasielec MS, Buckles VD, Cairns $\mathrm{NJ}$ et al (2016) Neurological manifestations of autosomal dominant familial Alzheimer's disease: a comparison of the published literature with the Dominantly Inherited Alzheimer Network observational study (DIAN-OBS). Lancet Neurol 15:1317-1325. https://doi.org/10.1016/S1474-4422(16)30229-0

9. Xi Z, Zinman L, Moreno D, Schymick J, Liang Y, Sato C et al (2013) Hypermethylation of the CpG island near the G4C2 repeat in ALS with a C9orf72 expansion. Am J Hum Genet 92:981-989. https://doi.org/10.1016/j.ajhg.2013.04.017

10. Zhang M, Tartaglia MC, Moreno D, Sato C, McKeever P, Weichert A et al (2017) DNA methylation age-acceleration is associated with disease duration and age at onset in C9orf72 patients. Acta Neuropathol 134:271-279. https://doi.org/10.1007/ s00401-017-1713-y

Publisher's Note Springer Nature remains neutral with regard to jurisdictional claims in published maps and institutional affiliations. 